\title{
Paper
}

\section{Low Profile UWB Frequency Selective Surface based Antenna}

\author{
Rabia Yahya ${ }^{\dagger}$, Akira Nakamura ${ }^{\dagger}$ (member) and Makoto Itami $^{\dagger}$ (member)
}

\begin{abstract}
In this paper, we present the design of a new ultra-wideband (UWB) frequency selective surface (FSS) based antenna, by using a wide stop-band FSS as a backing reflector for an UWB monopole antenna. A new single layer FSS, with UWB stop-band response and a reflection phase varies linearly with frequency, is firstly designed and thereafter, it is integrated with an UWB electric monopole. The studies of the behavior of the proposed antenna reveal that a unidirectional radiation with a quasi-constant gain of an average of $9 \mathrm{dBi}$ and a compact profile of $\lambda / 10$ are achieved. The features of the proposed UWB FSS and its based antenna make them good candidates for integration with low profile broadband printed circuits and UWB applications.
\end{abstract}

Keywords: Single-layer FSS; UWB FSS; FSS based antennas.

\section{Introduction}

During the last decades, an immense research has been devoted to developing the use of UWB technology in a number of different ways to significantly benefit of the attractive merits that this technology can offer. The interesting features of UWB technology, such as wide bandwidth, high data rate, interference mitigation and low transmission power, can optimize the performance of the existing systems and open the doors widely for new applications. Designing antennas meet the requirements of UWB applications is among the hot topics in the research field of UWB that have gained a lot of attention and attracted many research groups. Consequently, many antennas with UWB operation have been proposed ${ }^{1)-4)}$. Although the maximum effective isotropic radiation power (EIRP), of $-41.3 \mathrm{dBm} / \mathrm{MHz}$ imposed by UWB regulatory ${ }^{5)}$, can be satisfied with less power consumption through the employment of directional antennas; the most proposed UWB antennas are omnidirectional which provide the type of radiation needed by the applications where the users' locations are not predefined such as communication systems but also a directional radiation is more suitable for other applications such as radar and media applications. To make up the shortfall of UWB directional antennas especially with compact and planar structures, which is needed to facilitate their integration Received June 2, 2016; Revised August 10, 2016; Accepted September 5,2016

$\dagger$ Department of Applied Electronics, Tokyo University of Scienc (Tokyo, Japan) with microwave printed circuits and their fabrication using printed circuit board (PCB) technology, directional antennas based on various planar technologies have been reported ${ }^{6)-8)}$. These antennas used ground planes of different shapes to confine the radiation and make it directional. Although the shaped ground planes could serve the purpose of improving the directivity, the direction of the radiation was kept in the azimuth planes of the proposed antennas structures. Which may not be preferable for the applications that require the maximum radiation to be directed in the plane perpendicular to the antenna's plane (elevation plane). For providing antennas with such radiation and satisfying other requirements, such as broad bandwidth and high gain, different types of reflectors have been proposed from flat, conical metal reflectors ${ }^{9)}$ pass by EBG reflectors ${ }^{10) 11)}$ to FSS reflectors ${ }^{12)-14)}$. In addition to the ability of these reflectors to act as gain enhancers and stabilizers, they can decouple the mounted radiator from the surrounding objects that can damage its characteristics, which is of great interest for applications such as vehicular applications where the performance of used antennas can be affected by the vehicles bodies. In ${ }^{13) 14)}$, multilayer FSSs with stop-band responses have enabled different omnidirectional UWB antennas to have an improved gain across the UWB band, which proves that an FSS with an UWB stop-band response can serve as an UWB reflector especially when it can provide linear reflection phase over the UWB band. However, those proposed reflectors are multilayer FSSs where multiple spaced 
FSSs are needed, the thing that requires a large space and increases the error possibility by complicating the fabrication. All these disadvantages can be overcome by offering a single-layer FSS can serve as an UWB reflector. Therefore; in this paper, we present the design of a single-layer FSS can provide a stop-band response over the entire UWB band with linearly varied phase to own the ability to enhance the gain of planar monopole antennas in a gradual manner, which cannot be achieved by conventional flat metallic reflectors. The proposed unit cells cover one side of a single dielectric substrate with a compact cell size of $8 \mathrm{~mm} * 8 \mathrm{~mm}$. Thereafter, the proposed FSS and an UWB electric monopole are combined and redesigned to achieve UWB low profile antenna with a high quasi-constant gain. The operating bandwidth of our proposed antenna is wider, and its gain is higher and more stable than the ones reported in 10)-13). And its size is more compact than the ones proposed in 11)13)14). It worth to mention that the design and analysis process of the proposed FSS were performed using CST Microwave Studio 2015. The next section treats in details the design guide of the proposed FSS followed by the design process of the proposed FSSbased antenna using parametric studies to provide the essential design information. After that, the main results that describe the performance of the proposed antenna are presented and discussed.

\section{FSS Design Process}

The main idea of the proposed FSS's unit cell design is to combine two resonant elements, each one of them can exhibit a stop-band response over a part of the UWB band so that the resulting FSS can act as a stop-band filter over the entire UWB band, which is our design purpose. Stop-band response in the meaning that the incident waves are mostly reflected by the FSS with a transmission magnitude below $-10 \mathrm{~dB}$. A square loop element and a circular ring element have been chosen due to their ability to resonate at adjacent frequencies included in the UWB band. In order, to determine the design parameters that fulfill the objective of the desired adjacent resonance frequencies as well as compact size unit cells, the composed elements have been analyzed extensively. The parametric studies performed to investigate the behavior of the square unit cell revealed that increasing the length of the square loop, reduces its resonance frequency and narrows the width of its stopband. Although the internal length ( $\operatorname{Lin}$ ) of the square loop has similar effects as the length of the external square (Lout), when it is decreased, the lower frequency of the stopband doesn't change, which can compensate the effect of increasing the external length while reducing the resonance frequency. The effects of varying the both parameters Rout and Rin which denote respectively the outer and inner radiuses of the ring showed that the ring responds to the variation of its parameters in a similar way to the square loop. Increasing the outer radius leads to the reduction of the resonance frequency and the width of the stop-band. In contrast, reducing the inner radius moves the resonance frequency highly with a weak effect on the lower edge of the stop-band. The widest bandwidth is achieved when the two elements are connected. Otherwise, the frequency gap between the two resonance frequencies will be increased. The structure of the proposed unit cell is shown in Fig.1. It is printed on a high dielectric constant substrate of 10.2 and a thickness of $0.635 \mathrm{~mm}$. Based on the results of parametric studies of the composed elements and taking into consideration, the fact that the two elements must be connected to achieve a wide stop-band, which means that the outer radius of the ring should be superior or equal to the half of the internal length of the square loop, the final parameters of the unit cell of the proposed UWB FSS have been chosen and they are given in Table.1, where $\mathrm{g}$ is the gap width between the cells. Fig.2 illustrates the computed transmission coefficient of the proposed FSS along with its components, considering that the composed elements are printed on the same substrate with the same value of $\mathrm{g}$, to emphasize the main design guide of UWB FSSs with combined components. The main resonance frequency of the resulting FSS is at the intersection of

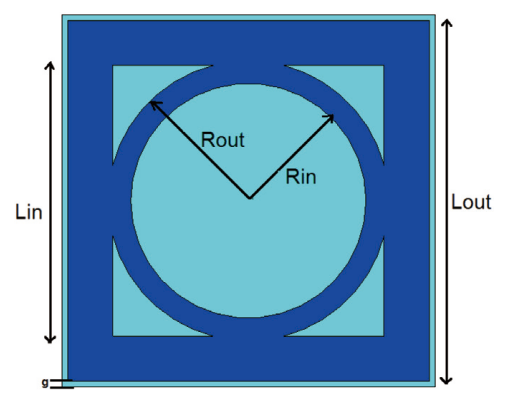

Fig.1 Structure of the proposed unit cell.

Table.1 Dimensions of the proposed unit cell (in $\mathrm{mm}$ ).

\begin{tabular}{|l|c|c|c|c|}
\hline $\mathrm{L}_{\text {out }}$ & $\mathrm{L}_{\text {in }}$ & $\mathrm{R}_{\text {out }}$ & $\mathrm{R}_{\text {in }}$ & $\mathrm{g}$ \\
\hline 7.75 & 6.25 & 3.225 & 2.725 & 0.25 \\
\hline
\end{tabular}




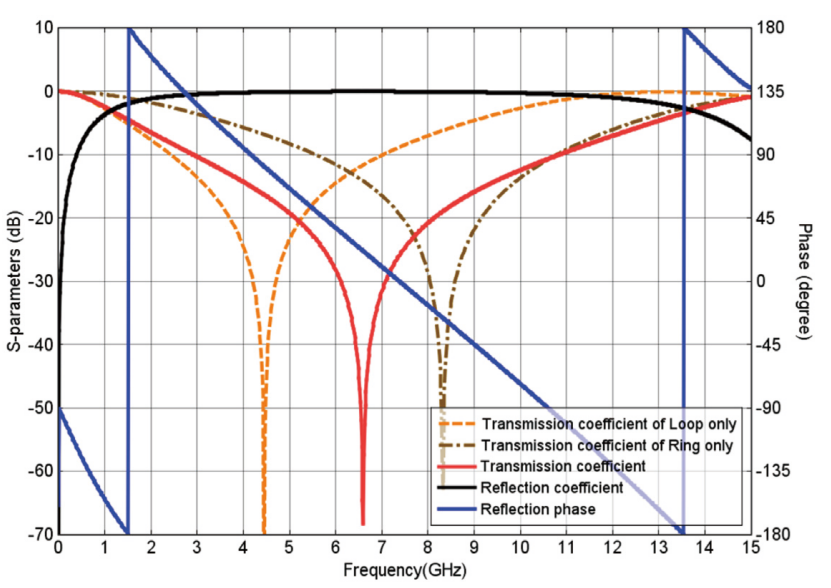

Fig.2 Reflection and transmission coefficients of the proposed FSS.

the transmission coefficients of its components, and its lower frequency limit is a little bit higher than that of its largest component in a contrary manner to its upper frequency limit which coincides with that of its smallest component. The reflection coefficient in Fig. 2 proves the ability of the proposed FSS to act as a stop-band filter over a wide-band from 3 to $11 \mathrm{GHz}$, which includes the entire UWB band with a reflection magnitude of $0 \mathrm{~dB}$ and a transmission magnitude inferior to $-10 \mathrm{~dB}$ and less than $-65 \mathrm{~dB}$ at the main resonance frequency around $6.5 \mathrm{GHz}$. The computed reflection phase of the FSS is linearly decreased with frequency, and it has zero value around $7 \mathrm{GHz}$. The linearity of the reflection phase of the proposed FSS is satisfied across the whole band from 2 to $13 \mathrm{GHz}$. This special feature extends the recommended applications of the proposed FSS to include pulsed systems where a linearly varied phase is highly required.

\section{UWB FSS with UWB monopole}

The plane wave analysis is the most common way to study and emphasize the performance of frequency selective surfaces where the incident waves are considered planar with incident angles around the normal on the plane of the FSS. Although this analysis method is useful for most of the applications that use FSSs as random and space filters, it is not that much useful when the FSSs are wanted to be integrated into the near-field zone of an antenna. However, several references used the planar approach to give a design guide for FSS-based antennas ${ }^{13)}{ }^{15)}$. On the other hand, ${ }^{12)}$ showed that the interactions between antennas and FSSs could not be sufficiently addressed without a full-wave analysis of the actual antenna structure in the presence of the FSS. Therefore, in this paper, instead of using FSSs to

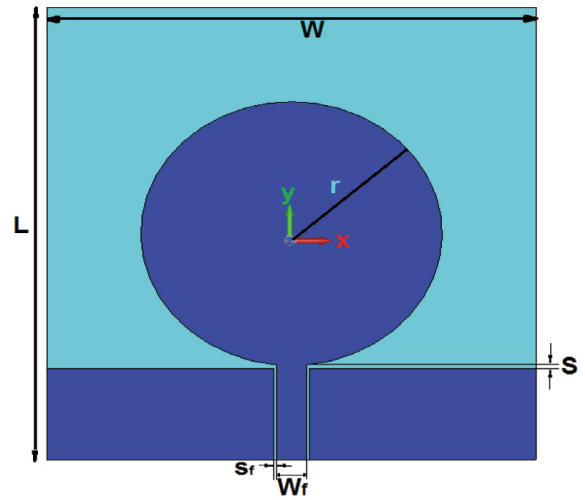

(a) UWB antenna [1]

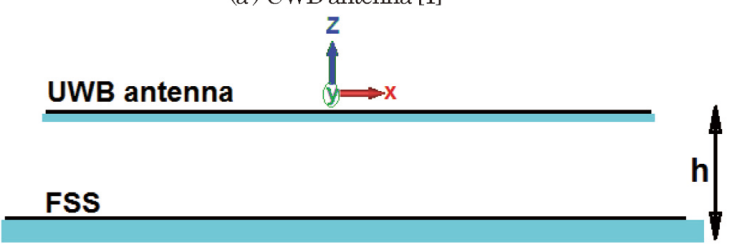

(b) Configuration of the UWB antenna installed above the proposed FSS.

Fig.3 UWB antenna with the proposed UWB FSS.

enhance the bandwidth of narrowband radiators as in 12) or using FSS based reflectors to improve the gain of predesigned UWB radiators as in ${ }^{12)-13)}$, we follow an approach gathers the best of all, where an UWB radiator along with a new single layer FSS are designed together to obtain an UWB, low profile, and high quasi-constant gain antenna. The used radiator is the antenna proposed in 1). The structure of this antenna is a circular disc fed by a CPW $50 \Omega$ line printed on a substrate of a dielectric constant of 3 and a thickness of $1.6 \mathrm{~mm}$ and the dimensions of the CPW line are $\mathrm{wf}=4 \mathrm{~mm}$ and $\mathrm{sf}=$ $0.33 \mathrm{~mm}$, where the former is the width of the main line and the latter is the gap between the main line of the CPW and the ground plane and $\mathrm{sf}=0.3$ is the slot gap between the circular disc and the ground plane, as shown in Fig.3.a. The UWB CPW-fed antenna was installed at distance $h$ above the proposed FSS, as indicated in Fig.3.b. Then, the new current distribution on the source, resulted by the integration of the FSS, was studied and analyzed to achieve the desired performance. As a result, the parameters of the both structures were changed. The structure of the proposed FSS-based antenna is illustrated in Fig.4. The feeding CPW line was tapered, and its new parameters are indicated in Fig.4, where; "Wt" is the width of its narrower side that touches the circular disc and "ls" is the length of the tapered part of the CPW line. This modification of the feeding line is necessary to compensate some of the effects of the FSS on the matching band of the source antenna especially that around the tapered area, the current is maximum which 


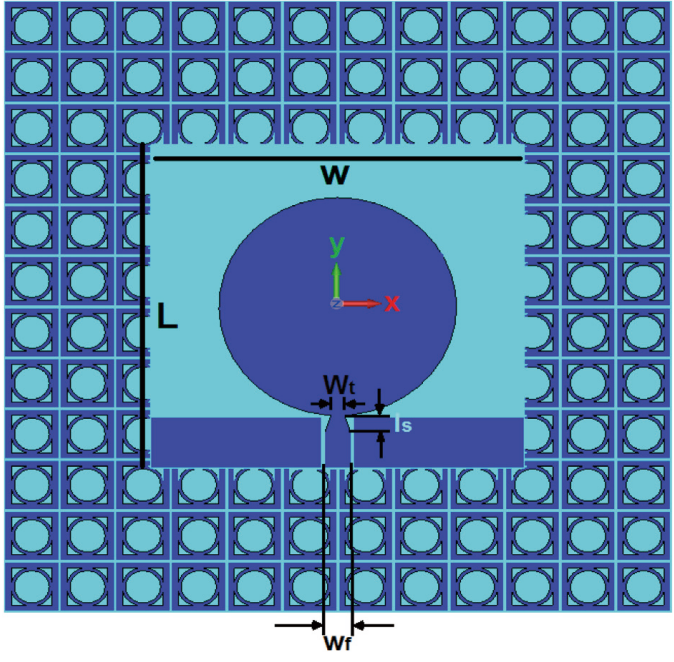

Fig.4 Proposed structure of UWB FSS based antenna

indicates that this area has a big effect on the matching band of the antenna.

In addition to the main parameters that control the matching band of the monopole antenna, such as r, w, and $\mathrm{s}$, the new parameter $\mathrm{h}$ has a big effect on the matching band as it can be seen in Figure.5.a, which shows the reflection coefficient of the proposed antenna for different values of $\mathrm{h}$ and also shows that of the reference antenna. Comparing with the reference antenna, we can see that the FSS affects the matching band of the source antenna also we can derive that as $h$ increases, the bandwidth of the antenna increases. However, one should pay attention that the parameter $h$ sets the profile of the proposed antenna hence, the minimum value of $h$ that gives the necessary bandwidth should be chosen. Also, this parameter influences the radiation behavior of the antenna because it adds a phase shift to the reflected waves from the FSS. This is shown clearly in Fig.5.b where the peak gain of the proposed antenna, across the UWB band, is computed at different values of $h$. From which, we can notice that the gain changes differently according to the frequency which can be explained by the fact that the added phase shift is a function of the frequency. It worth to mention that the peak gain is the maximum gain computed at each frequency and not at a special direction and that because the maximum direction of the used radiator is aligned with frequency. In addition to its effects on the provided performance, the size of the FSS determines the overall size of the antenna. Therefore, a deep study of the effect of the size of the installed FSS is performed and its results are shown in Fig.6. Which reveals that the matching band of the antenna is mainly affected by the part of the FSS that is located directly under the

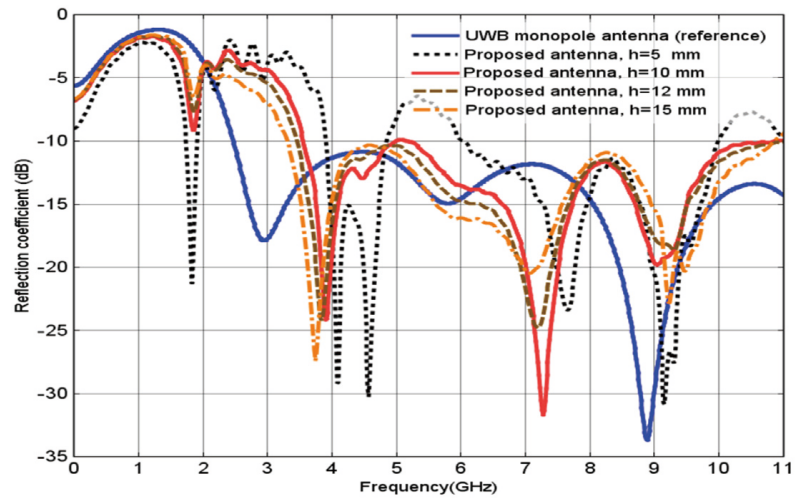

Fig.5.a Reflection coefficient for different values of $h$.

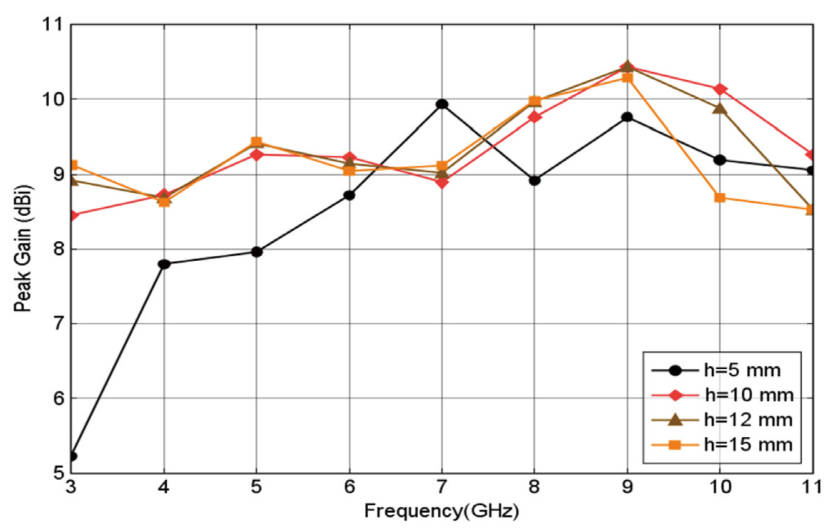

Fig.5.b Gain for different values of $h$.

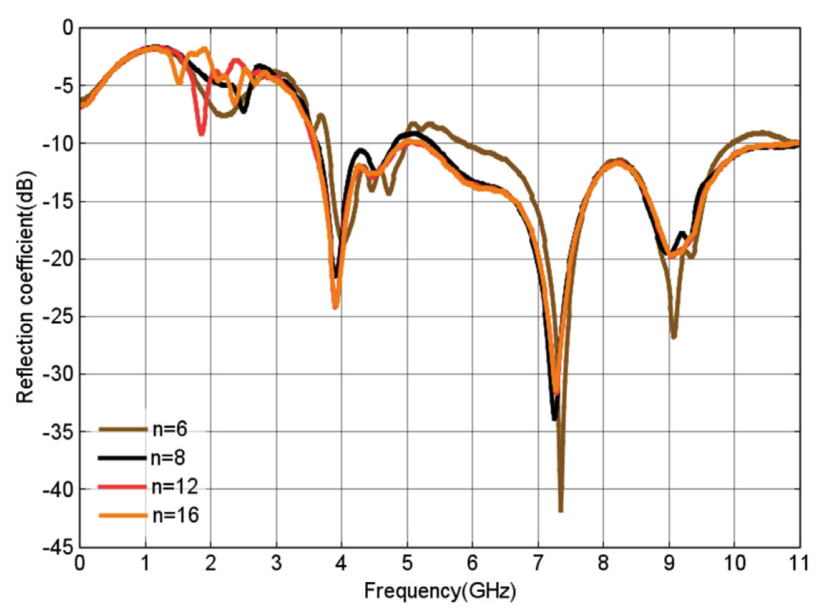

Fig.6.a Reflection coefficient for different number of cells.

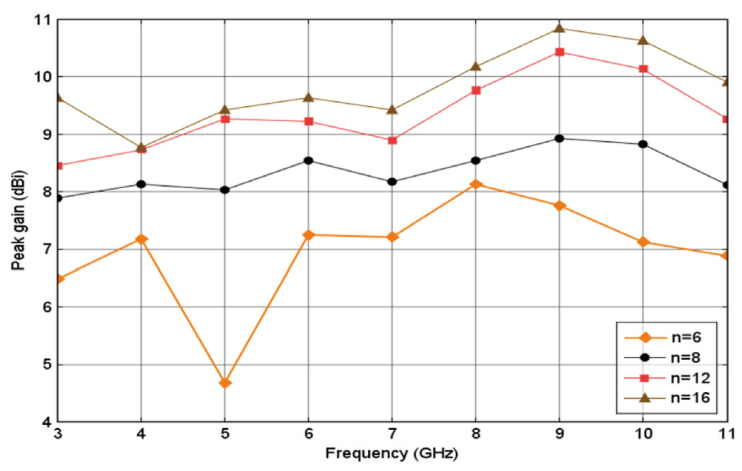

Fig.6.b Gain for different number of cells. 
source as can be noticed in Fig.6.a that contains the reflection coefficient of the antenna for different numbers of cells (n), which models the size of the FSS. In other words, when the FSS is larger than the source, the bandwidth of the antenna is almost independent of the FSS size. However, the size of the FSS affects mainly the radiation behavior of the antenna, which is shown in Fig.6.b by means of a parametric study of the antenna peak gain. From which, we can notice that as the number of cells increases, the gain is increased over the entire UWB band. Finally, the parameters of the structure were optimizing, using CST-MWS, to achieve UWB operating band and a quasi-constant gain over it with a minimum variation possible.

\section{Final Results of FSS based Antenna}

The final optimized dimensions of the proposed antenna are given in Table.2. Fig.7 indicates the computed reflection coefficient of the proposed antenna, which shows that a reflection magnitude inferior to $-10 \mathrm{~dB}$ is achieved along the band from $3.5 \mathrm{GHz}$ to $10.6 \mathrm{GHz}$. This matching band is obtained for overall profile thickness of $10 \mathrm{~mm}$ which is around $\lambda / 10$ at the lower operating frequency. The matching band can be broadened, as in Fig.5.a, by choosing a larger value of $h$. Regarding the radiation behavior, the radiation patterns computed at $6 \mathrm{GHz}$ for the UWB antenna in ${ }^{1)}$ and the proposed antenna, in $\mathrm{H}$-plane and E-plane, are illustrated in Fig.8. The radiation pattern of the UWB monopole antenna is omnidirectional in H-plane where the antenna acts as a conventional monopole antenna, and it is bidirectional in E-plane while the proposed antenna has a reduced back radiation in the both planes hence, an increased directivity. The peak gain of the proposed antenna and the monopole antenna in ${ }^{1)}$, across the frequency, are indicated in Fig.9. It is evident that the proposed antenna's gain is much higher, across the UWB band, than that of the monopole antenna. The amount of enhancement is varied across the band from $6.5 \mathrm{dBi}$ at $3 \mathrm{GHz}$ to $3.5 \mathrm{dBi}$ at $10 \mathrm{GHz}$, which led to a quasi-constant gain with a maximum variation of $0.7 \mathrm{dBi}$ across the entire UWB band. Different factors such as the linearly decreased reflection phase of the proposed FSS and the small distance between the FSS and the radiator, which cannot be obtained using a flat metallic reflector, are contributed to achieving the resulted high quasi-constant gain. As a result, a low profile planar UWB antenna with enhanced quasi-constant gain is obtained.
Table.2 Dimensions of the proposed FSS-based antenna (in $\mathrm{mm}$ ).

\begin{tabular}{|l|c|c|c|c|c|c|c|c|c|c|c|}
\hline Lout & $\mathrm{L}_{\text {in }}$ & $\mathrm{R}_{\text {out }}$ & $\mathrm{R}_{\text {in }}$ & $\mathrm{g}$ & $\mathrm{L}$ & $\mathrm{W}$ & $\mathrm{r}$ & $\mathrm{Wt}$ & $\mathrm{hs}$ & $\mathrm{n}$ & $\mathrm{h}$ \\
\hline 8 & 6 & 3.1 & 2.5 & 0.25 & 52 & 55 & 17.5 & 2 & 3 & 12 & 10 \\
\hline
\end{tabular}

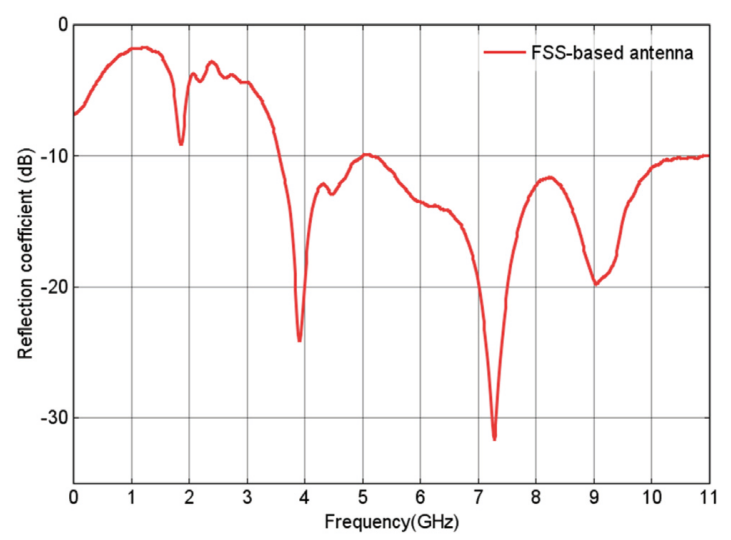

Fig.7 Reflection coefficient of the proposed antenna.

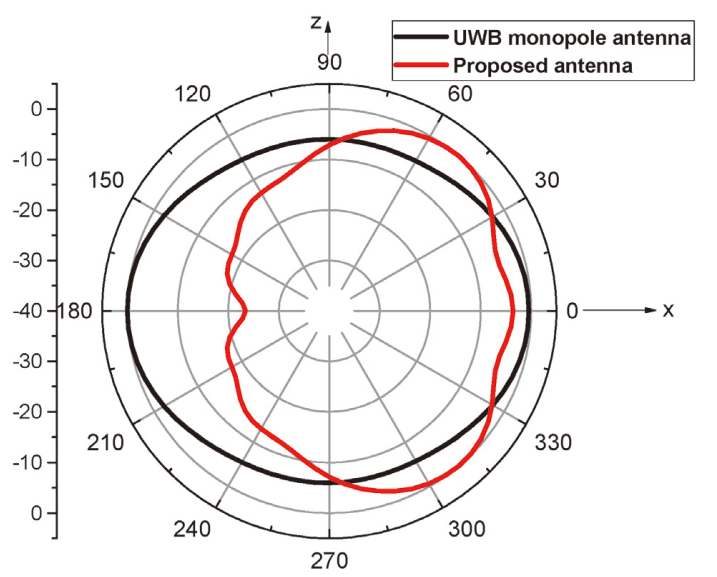

(a) In H-plane (xz)

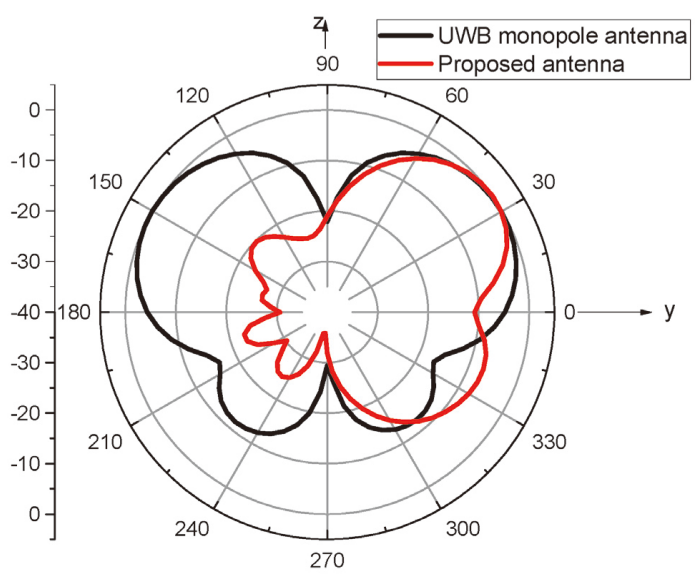

(b) In E-plane (yz)

Fig.8 Radiation Patterns of the monopole antenna ${ }^{1)}$ and the proposed antenna.

\section{Conclusion}

The main objective of this paper is to design an UWB low profile planar antenna with a high quasi-constant 


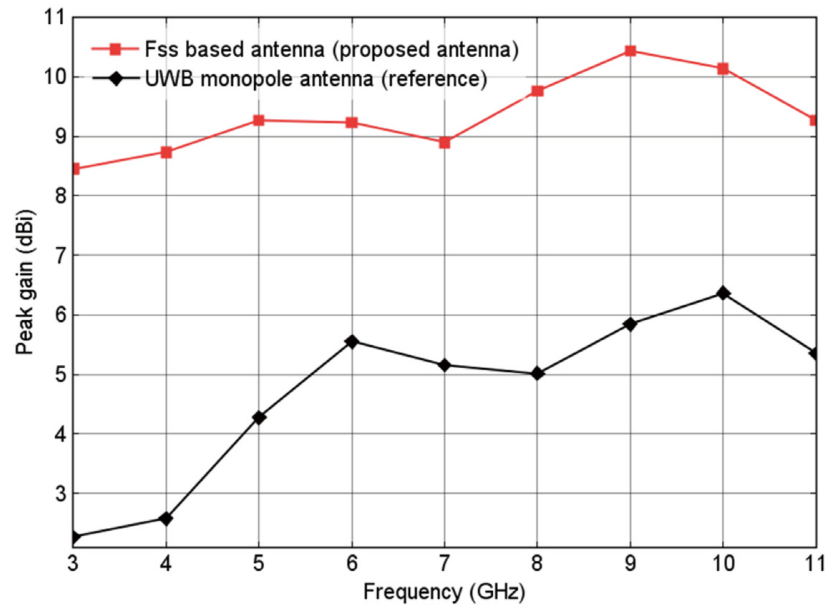

Fig.9 Peak gain for the proposed antenna and the one proposed in ${ }^{1}$.

gain. Therefore, a new single-layer UWB FSS with the ability to enhance the gain of UWB monopole antennas has been designed. Thereafter, it was integrated with an UWB electric monopole as an UWB reflector. The design process of the proposed FSS and its based antenna has been presented and discussed to give insights into the operation mechanism. The results that prove the concept of the designs have also been shown. The compact size and light profile of the proposed designs and their proved performance make them suitable for UWB applications and good candidates for integration with broadband compact printed circuits. The quasi-constant high gain of the proposed antenna can increase its coverage range with respect to UWB regulation. It is also recommended for the applications used in environments that can affect antennas performance because the antenna's reflector can protect its performance from the surroundings.

\section{References}

1) J. Liang, L. Guo, C.C. Chiau, X. Chen and C.G. Parini: "Study of CPW-fed circular disc monopole antenna for ultra wideband applications", IEE Proc.-Microw. Antennas Propag, vol. 152, No.6 (Dec. 2005)

2) T.A. Denidni and M.A. Habib: "Broadband printed CPW-fed circular slot antenna", Electronics Letters., 42, 3 (Feb. 2006)

3) R. Yahya and Tayeb A. Denidni: "Design of a new Ultra-Wideband Antenna with Dual Polarization operation", International Journal on Communications Antenna and Propag., 3, 5, pp.245-248 (Oct. 2013)

4) Shun-Shi Zhong, Xian-Ling Liang and W. Wang: "Compact Elliptical Monopole Antenna with Impedance Bandwidth in Excess of 21:1", IEEE Trans. Antennas Propag., 55, 11, pp.3082-3085 (Nov. 2007)

5) First Report and Order, Revision of Part 15 of the Commission's Rules Regarding Ultra-Wideband Transmission Systems, Federal Communications Commission, ET Docket 98-153, FCC 02-48 (Feb. 2002)

6) S. Wang, X.D. Chen and C.G. Parini: "Analysis of Ultra-wideband antipodal Vivaldi antenna design", Antennas and Propagation Conference., pp.129-132 (Apr. 2007)
7) A. Locatelli, D. Modotto, F.M.Pigozzo, S.Boscolo, E. Autizi, C. De Angelis, A.-D. Capobianco, M. Midrio: "Highly directional planar Ultra Wide Band antenna for radar applications", European Microwave Integrated Circuit Conference, EuMIC 2007., pp.14211424 (Oct. 2007)

8) M. Mokhtaari, J. Bornemann: "Directional Ultra-Wideband Antennas in Planar Technologies", European Microwave Conference, pp.885-888 (Oct. 2008)

9) Zhao, J. Y., Zhang, Z. Y., Liu, N. W., Fu, G., \& Gong, S. X: "Wideband Unidirectional Bowtie Antenna with Pattern Improvement.", Progress In Electromagnetics Research Letters, Vol.44, pp.119-124 (2014)

10) L. Akhoondzadeh-Asl, D.L. Kern, P.S. Hall, D.H. Werner: "Wideband dipoles on electromagnetic bandgap ground planes", Antennas and Propagation, IEEE Transactions on, 9, 55, pp.24262434 (Sept 2007)

11) Q. Wu. Ronghong Jin, J. Geng and D. Su: "On the performance of printed dipole antenna with novel composite corrugated-reflectors for low-profile ultrawideband applications", Antennas and Propagation, IEEE Transactions on, 58, 12, pp.3839-3846 (Dec. 2010)

12) A. Foroozesh and L. Shafai: "Investigation into the application of artificial magnetic conductors to bandwidth broadening, gain enhancement and beam shaping of low profile and conventional monopole antennas", Antennas and Propagation, IEEE Transactions on, 59, 1, pp.4-20 (Jan 2011)

13) Y. Ranga, L. Matekovits, K.P. Esselle and A.R. Weily: "Multioctave frequency selective surface reflector for ultrawideband antennas", Antennas and Wireless Propagation Letters, IEEE, Vol.10, pp.219$222(2011)$

14) Y. Ranga, L. Matekovits, A.R. Weily and K.P. Esselle: "A Constant gain Ultra-Wide band Antenna with A Mlti-Layer Frequency Selective Surface", Progress In Electromagnetics Research Letters, Vol.38, pp.119-125 (2013)

15) F. Yang and Y. Rahmat-Samii: "Electromagnetic band gap structures in antenna engineering", UK: Cambridge university press (2008)

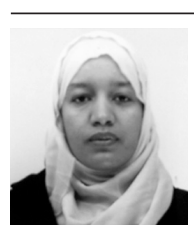

Rabia Yahya was born in Chinguitti, Mauritania, in 1989. She received M .Sc. Degrees in telecommunication from Instutit national de la recherche scientifique (INRS), Montreal, Canada, in 2011. In 2013, she started as a research student in Tokyo University of Science. Currently, she is a Ph.D. candidate in Dept. of Applied Electronics, Tokyo University of Science. Her current research interests include ultra-wideband (UWB) antennas, frequency selective surface (FSS) and Reconfigurable antennas. She is a member of IEEE.

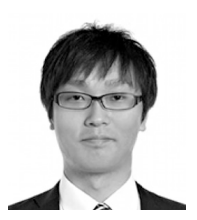

Akira Nakamura received his B.E. and M.E. degree in Electronics Engineering from Tokyo University of Science, Japan, in 2009 and 2011, respectively. He received Ph.D. degree of Electronics Engineering from Tokyo University of Science in 2013. $\mathrm{He}$ is an assistant professor at Dept. of Applied Electronics, Tokyo University of Science. His current research interests include wireless communication and broadcasting using OFDM modulation. He is a member of ITE, IEICE and IEEE.

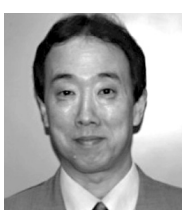

Makoto Itami received B. Eng., M. Eng. and Dr Eng. degrees from the University of Tokyo, in 1984, 1986 and 1989, respectively. In 1989, he joined the Department of Applied Electronics at Tokyo University of Science, Japan, and since 2008. He has been a Professor. His research interests are in the fields of communication systems and digital signal processing. $\mathrm{He}$ is a member of ITE, IEICE and IEEE. 